\title{
Characterization of the Superhyperfine Interaction in ${ }^{171} \mathbf{Y b}: \mathbf{Y V O}_{4}$
}

\author{
Yan Q. Huan ${ }^{1,2}$, Jonathan M. Kindem ${ }^{1,2}$, John G. Bartholomew ${ }^{1,2}$, and Andrei \\ Faraon $^{1,2}$ \\ ${ }^{1}$ Kavli Nanoscience Institute and Thomas J. Watson, Sr., Laboratory of Applied Physics, California Institute of Technology \\ Pasadena, California 91125, USA \\ ${ }^{2}$ Institute for Quantum Information and Matter, California Institute of Technology, Pasadena, California 91125, USA \\ yhuan@caltech.edu
}

\begin{abstract}
We computationally characterize the superhyperfine energy structure of ${ }^{171} \mathrm{Yb}: \mathrm{YVO}_{4}$ and compare predicted holeburning spectra and coherence times with experimental data. Our simulation can help optimize coherence times for ensemble-based quantum memories and single-ion qubits. (C) 2019 The Author(s)
\end{abstract}

OCIS codes: 160.5690 (Rare-earth-doped materials), 270.5585 (Quantum information and processing)

Rare-earth ions in crystals are a promising platform for quantum memories and quantum information processing in the solid state [1] because they possess many desirable properties such as long optical and spin coherence times [2]. In particular, ${ }^{171} \mathrm{Yb}^{3+}$ ions are attractive because their electronic and nuclear spins of $1 / 2$ allow for coupling to both optical and microwave photons while maintaining a simple energy level structure. In addition, their coherence properties have recently been studied and show promise for quantum memories and optically addressable single rare-earth-ion qubits [3]. One important aspect of such systems is the superhyperfine interaction between the rare-earth ion and host nuclei, which could be used to address the host nuclear spins to store quantum information in long-lived superhyperfine states, but at the same time is a significant contribution to decoherence of the optical and spin transitions at cryogenic temperatures [2].

In this work, we present a computational characterization of the energy level structure of the ${ }^{2} F_{7 / 2}(0) \leftrightarrow$ ${ }^{2} F_{5 / 2}(0)$ transition of ${ }^{171} \mathrm{Yb}^{3+}$ in $\mathrm{YVO}_{4}$ including the superhyperfine interaction with the ion's nearest neighbor spins. We demonstrate the utility of this model by predicting spectral holeburning spectra, which are successfully matched against experimental results. Extending on this, we use our model to search for accessible optical $\Lambda$-systems at zero field that utilize the superhyperfine levels. We further explore the superhyperfine interaction by modeling neighbor spin flip-flops that cause decoherence of the $\mathrm{Yb}^{3+}$ ion and demonstrate a first-principles calculation of the coherence lifetimes for both optical and nuclear spin transitions. Our predicted coherence times agree with experimental results from two-pulse photon echo measurements to within a factor of two with no fitting parameters. The predictive power of our simulation can potentially aid in discovering accessible superhyperfine states for future long-lived quantum memories as well as help optimize coherence times for ensemble-based quantum memories and single-ion qubits.

The energy level structure of the $\mathrm{Yb}^{3+}$ ion was computed by considering the superhyperfine interactions with the nearest neighbor spins in the host crystal as well as the Zeeman and hyperfine terms. The effective spin Hamiltonian was solved using a Python program to predict the energy spectrum (as shown in Fig. 1), eigenstates, and transition amplitudes for arbitrary strengths and orientations of the magnetic field. By applying perturbation theory, we estimated the magnitude of the superhyperfine broadening for each of the four energy eigenstates at zero field, of which two have aligned electronic and nuclear $\mathrm{Yb}^{3+}$ spins $\{|\uparrow \uparrow\rangle,|\downarrow \downarrow\rangle\}$ and the other two have zero expectation value for the spins $\left\{\frac{1}{\sqrt{2}}(|\uparrow \downarrow\rangle \pm|\downarrow \uparrow\rangle)\right\}$. By considering $\sim 10000$ neighboring yttrium and vanadium atoms, we computed the maximum superhyperfine broadening at zero field for the nonzero-spin states to be about $110 \mathrm{MHz}$ and $45 \mathrm{MHz}$ for the ground and excited state respectively, and $1.3 \mathrm{MHz}$ and $45 \mathrm{kHz}$ for the ground and excited state respectively for the zero-spin states.

To verify the accuracy of the spin Hamiltonian simulations, we computed holeburning spectra by simulating an optical pumping experiment. Fig. 2 illustrates the simulated spectrum in orange against experimental results in black, where the pumped transitions are indicated in red in Fig. 1. In order to match the predictions with our experiments in which the laser was dithered over a range of $190 \mathrm{MHz}$ while optical pumping, the simulated holeburning spectrum was convolved with a rectangular window of that same frequency width as shown in blue. The simulation demonstrates good qualitative agreement with the experimental results.

As an application of our simulation, we searched for optical $\Lambda$-systems involving superhyperfine levels at zero B field as the Yb:YVO system exhibits zero first-order Zeeman (ZEFOZ) transitions at zero field. We considered the effects of the 6 nearest neighbors, and searched for $\Lambda$-systems involving superhyperfine levels where both 


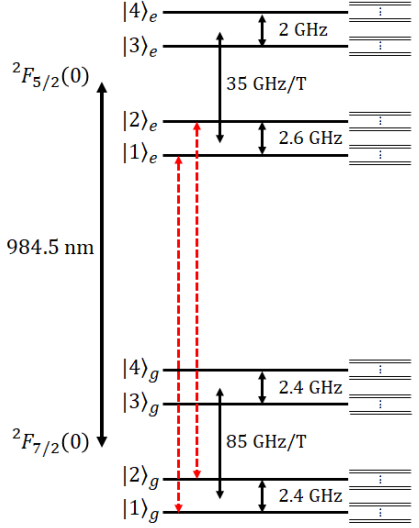

Fig. 1: Energy level diagram for ${ }^{171} \mathrm{Yb}: \mathrm{YVO}$ in the limit of large $\mathbf{B}$ along the crystal $c$-axis with magnitudes of the Zeeman and hyperfine splitting indicated. Each state is further split into a multitude of closespaced states by the superhyperfine interaction. The red dotted transitions are pumped for spectral holeburning measurements.

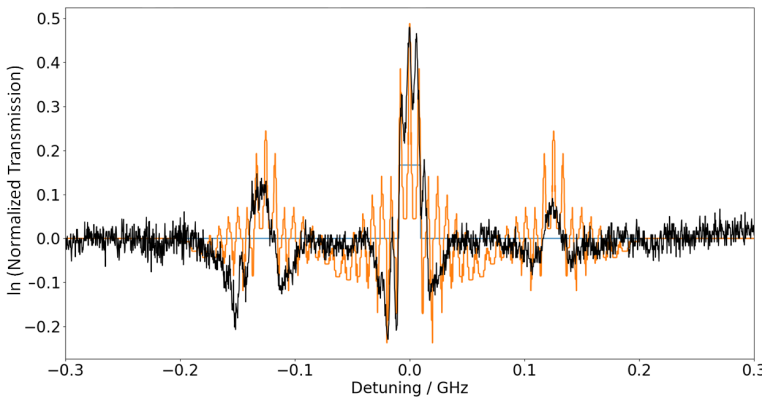

Fig. 2: Theoretical (orange) and experimental (black) holeburning spectrum of ${ }^{171} \mathrm{Yb}: \mathrm{YVO}$ in a field at an angle of $19.4^{\circ}$ from the crystal $c$-axis and the light polarized along the $a$-axis. Theoretical predictions were convolved against a $190 \mathrm{MHz}$ rectangular window (blue) to model the holeburning laser frequency dither.

optical transitions were allowed and the ground state splitting was at least $1 \mathrm{kHz}$. We found over $200 \Lambda$-systems with $\mathbf{E}$ field polarized along $c$ that satisfied the above requirements, but the maximum energy difference between the two ground states in these systems was only $21 \mathrm{kHz}$. This indicates that the superhyperfine interaction alone is insufficient to split states sufficiently at zero-field for them to be optically accessible, and suggests that ZEFOZ states at higher fields may be more a promising option.

Finally, as the superhyperfine interaction and $\mathrm{Yb}$ - $\mathrm{Yb}$ spin flip-flop interactions are the primary sources of dephasing at sub-K temperatures in our system, we were able to use our simulation to predict coherence lifetimes for the optical and nuclear spin transitions of the ${ }^{171} \mathrm{Yb}$ ion. We applied perturbation theory on our energy eigenstates to obtain the transition sensitivity $\frac{\partial \omega}{\partial \mathbf{B}}$ and simulated spin flips of the neighboring atoms to estimate the $\mathbf{B}$ field fluctuations. Using these parameters, we estimated the resultant coherence times $[4,5]$ and compared against photon-echo and spin-echo decay measurements from two-pulse echo experiments [3]. Figs $3 \mathrm{a}$ and $3 \mathrm{~b}$ show a close correspondence of both the qualitative trend and numerical values between the predictions and experimental results. Additionally, the theoretical predictions were refined by allowing the spin flip rate of $\mathrm{Yb}$ and the magnetic fluctuations from neighbor $\mathrm{V}$ atoms to vary as the value of the former has not been measured while the latter depends on the exact radius of the "frozen core". This yielded a better fit as shown in yellow. We believe that our theoretical simulations can be helpful in exploring Yb:YVO and other materials to discover promising systems with long coherence times for future ensemble-based quantum memories and single-ion qubits.
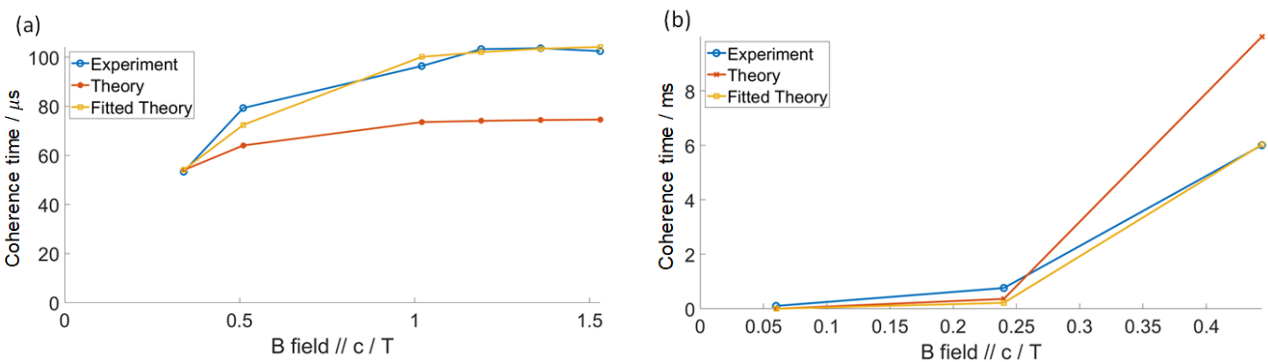

Fig. 3: (a) Photon echo and (b) nuclear-spin echo decay coherence lifetimes $T_{2}$ as a function of $\mathbf{B} \| c$ for $100 \mathrm{ppm}$ $\mathrm{Yb}$ doping at $650 \mathrm{mK}$ and $700 \mathrm{mK}$ respectively.

\section{References}

1. K. Heshami et al., "Quantum memories: emerging applications and recent advances," Journal of Modern Optics 63, 2005-2028 (2016).

2. M. Zhong et al., "Optically addressable nuclear spins in a solid with a six-hour coherence time," Nature 517, 177-180 (2015).

3. J. M. Kindem et al., "Characterization of ${ }^{171} \mathrm{Yb}^{3+}: \mathrm{YVO}_{4}$ for photonic quantum technologies," Physical Review B 98 (2018).

4. T. Böttger, C. W. Thiel, Y. Sun, and R. L. Cone, "Optical decoherence and spectral diffusion at $1.5 \mu \mathrm{m} \mathrm{in} \operatorname{Er}^{3+}: \mathrm{Y}_{2} \mathrm{SiO}_{5}$ versus magnetic field, temperature, and $\mathrm{Er}^{3+}$ concentration," Physical Review B 73 (2006).

5. M. Zhong, "Development of persistent quantum memories," Ph.D. thesis, The Australian National University, Canberra (2017). 